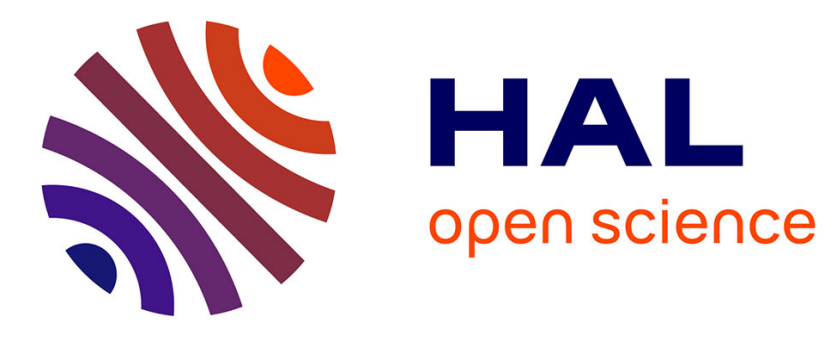

\title{
In situ break-junction sample holder for transmission electron microscopy
}

Santhana K. Eswara Moorthy, Gerald Le Goff, Michel Viret, Mathieu K

Kociak

\section{- To cite this version:}

Santhana K. Eswara Moorthy, Gerald Le Goff, Michel Viret, Mathieu K Kociak. In situ breakjunction sample holder for transmission electron microscopy. European Physical Journal: Applied Physics, 2013, 64, pp.31001. 10.1051/epjap/2013130365 . cea-01498654

\section{HAL Id: cea-01498654 https://hal-cea.archives-ouvertes.fr/cea-01498654}

Submitted on 30 Mar 2017

HAL is a multi-disciplinary open access archive for the deposit and dissemination of scientific research documents, whether they are published or not. The documents may come from teaching and research institutions in France or abroad, or from public or private research centers.
L'archive ouverte pluridisciplinaire HAL, est destinée au dépôt et à la diffusion de documents scientifiques de niveau recherche, publiés ou non, émanant des établissements d'enseignement et de recherche français ou étrangers, des laboratoires publics ou privés. 


\title{
In situ break-junction sample holder for transmission electron microscopy
}

\author{
Santhana K. Eswara Moorthy ${ }^{1, a, b}$, Gerald Le Goff ${ }^{2}$, Michel Viret ${ }^{2}$, and Mathieu Kociak ${ }^{1}$ \\ ${ }^{1}$ Laboratoire de Physique des Solides, CNRS, UMR 8502 Université Paris Sud, 91405 Orsay, France \\ 2 Service de Physique de l'Etat Condensé, URA CNRS 2464 CEA/DSM/IRAMIS/SPEC Orme des Merisiers, \\ 91191 Gif-sur-Yvette, France
}

Received: 2 August 2013 / Received in final form: 2 October 2013 / Accepted: 18 October 2013

Published online: 9 December 2013 - (C) EDP Sciences 2013

\begin{abstract}
In this article, we report on the design and construction of an in situ break-junction sample holder for transmission electron microscopy. The holder is based on the differential-screw mechanism. The technical details and a comprehensive consideration to all relevant critical issues surrounding the instrumentation procedure are presented. An application of the newly developed instrument is demonstrated using the example of a micro-scale gold wire. We also provide a detailed discussion on the challenges involved and the pitfalls to avoid in developing similar in situ holders.
\end{abstract}

\section{Introduction}

In situ experiments in transmission electron microscopes (TEM) offer enormous advantages by enabling direct observations and analyses of physical and chemical behaviors of materials at superior spatial-resolution. Such techniques allow probing materials properties to gain fundamental understanding as well as to study in-service behavior to develop materials technology. Over the past several decades, in situ sample holder capabilities have been developed primarily for heating [1], cryogenic [2], straining [3] and magnetizing [4] (Lorentz microscopy) studies. Another notable development in this domain is environmental TEM which allows studying chemical reactions in situ by controlling the gas pressure in the sample chamber [5]. More recently, with the advent of nanoscale positioning capabilities offered by piezoelectric ceramics, new capabilities such as nanoindentation [6] as well as in situ STM/AFM holders have been added to the in situ capabilities of electron microscopes $[7,8]$.

In this article, we report our work on instrumentation development of in situ mechanically controlled break-junction (MCBJ) sample holder for TEM. MCBJ have been previously used for ex-situ studies of electron-transport properties to understand quantum conductance [9]. Generally speaking, it is composed of a nanowire suspended on a flexible substrate. Upon bending

\footnotetext{
a e-mail: eswara@lippmann.lu

b Current address: Scientific Instrumentation Unit (UIS), Département Science et Analyse des Matériaux (SAM), Centre de Recherche Public - Gabriel Lippmann, 41, rue du Brill, L-4422 Belvaux, Luxembourg.
}

the substrate progressively using two counteracting forks, the suspended nanowire is stretched gradually until rupture. By constantly monitoring the current (I)-voltage (V) plot during the rupture, one can then analyze the electronic behavior of atomic scale nanojunctions. However, the most severe limitation of such ex-situ break-junction studies is that there is no direct access to ascertain the instantaneous structure and chemical composition of the nanowire. Hence, the observed properties cannot be accurately correlated to structure and chemistry.

Previously, in situ MCBJ for a scanning electron microscope (SEM) has been reported by Taychatanapat et al. [10]. However, direct implementation of such set-up for a TEM is non-trivial because of the overwhelming technical limitations, such as the narrow gap between the polepieces. Hence, in order to fully exploit the advantages of TEM, such a holder for TEM should be developed.

The objective of this work is, therefore, to overcome the aforementioned limitations in ex-situ studies by developing an in situ break-junction sample holder for TEM and thus enable a direct determination of structural and chemical information and the simultaneous evaluation of electronic properties. One of the significant advantages of the break-junction in situ TEM holder over other conventional in situ straining holders or the in situ STM-TEM holder is that the ruptured sample can be reconnected by relaxing the flexible substrate and the study can be repeated again and again in order to obtain enough statistical data on similar structures with exactly the same chemical composition.

In this article, we provide the technical design of the holder and strategies to overcome technical limitations. 

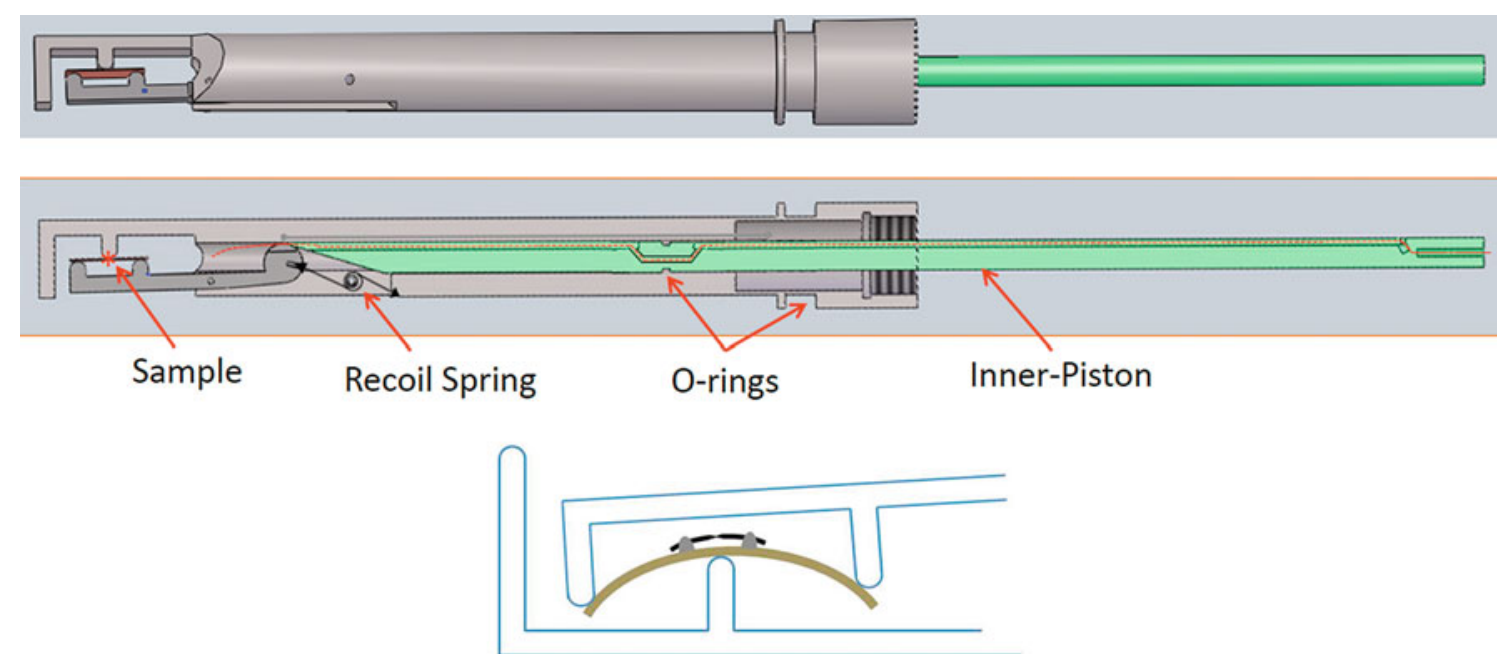

Fig. 1. (A) Schematic of the mechanical break-junction set-up adapted for in situ TEM. The inner-piston is shown in green. (B) Cross-sectional view of the assembly showing the relative orientation of the inner-piston to the mobile fork. A spring is indicated by the black arrow, between the inner-piston and the mobile fork. By advancing and retracting the inner-piston, the mobile fork can flex or relax the mounted sample. The red arrows indicate the positions of the O-rings. The trench on the inner-piston for copper wire feed-through is also visible. The position of the electron beam travelling perpendicular to the plane of view is indicated by red asterisk. (C) Enlarged schematic of the head of the MCBJ illustrating the configuration of the sample assembly. The gold filament (black) is suspended and attached using a commercial vacuum-epoxy, TorrSeal (grey), onto the flexible Kapton (brown) support.

We also provide a detailed discussion on the challenges involved and the pitfalls to avoid in developing similar in situ holders.

\section{Instrumentation development}

The in situ TEM sample holder described below was developed for a conventional TEM, Topcon 002B with a $\mathrm{LaB}_{6}$ thermionic-emission gun. The normal operating vacuum in the column is better than $5 \times 10^{-7}$ torr. The microscope was equipped with ultra-high resolution (UHR) objective lens pole-pieces with a nominal gap between the UHR pole-pieces of $4 \mathrm{~mm}$. The permissible thickness of the in situ holder is thus severely limited. Furthermore, any increase in the pole-piece gap deteriorates the spatial resolution due to less uniform magnetic field of the objective lens.

Another consequence of the limited pole-pieces gap is that the sample-tilt achievable is limited to $\pm 10^{\circ}$ about the sample-holder axis $(x)$. Tilting beyond this limit will lead to sample-holder touching the pole-pieces which could damage them. Tilt capabilities are necessary in order to overcome shadowing-effect that can be caused when substrates are not perfectly aligned with the optic-axis of the microscope. As an illustration, consider a typical substrate width of $1 \mathrm{~mm}$ with a wire suspended at a distance of $2 \mu \mathrm{m}$ from the center of the substrate. It is understandable that even a slight misalignment of the substrate, tilted off from the optic-axis by $4 \mathrm{mrad}\left(0.24^{\circ}\right)$ is sufficient to fully obscure the wire. Therefore, tilt-resolution, i.e., the step size between two consequent tilt-settings, is equally important as the total tilt-range available. Fortunately, the tilt-resolution reachable by the microscope used is around $0.1^{\circ}$. For other microscopes, this has to be taken into account while designing similar holders adapted to specific sample geometries.

\subsection{The outer tube and the inner piston}

A schematic diagram of the break-junction, as configured for the in situ TEM holder is shown in Figure 1. The corresponding image of the sample holder is shown in Figure 2. As briefly introduced earlier, two counteracting forks flex the substrate thereby gradually straining the suspended wire. One of the forks is a fixed extension of the sampleholder chassis while the other is movable using a lever based set-up. The movable fork can be used to flex or relax the sample by advancing or retracting the inner-piston, which is coupled to the external screw. Note the trench on the inner-piston shown in Figure 1B for copper wire feedthrough indicated by red dotted-line. This offers in situ electrical measurements capability.

\subsection{The differential-screw}

In order to accurately control the degree of flexing and relaxing of the sample, precise positioning of the innerpiston is imperative. We achieved such a fine positioncontrol by designing and implementing a differential-screw mechanism. Since the differential-screw mechanism is a rather standard technique in mechanical designing, only a brief description is provided below for the sake of completeness and clarity. 

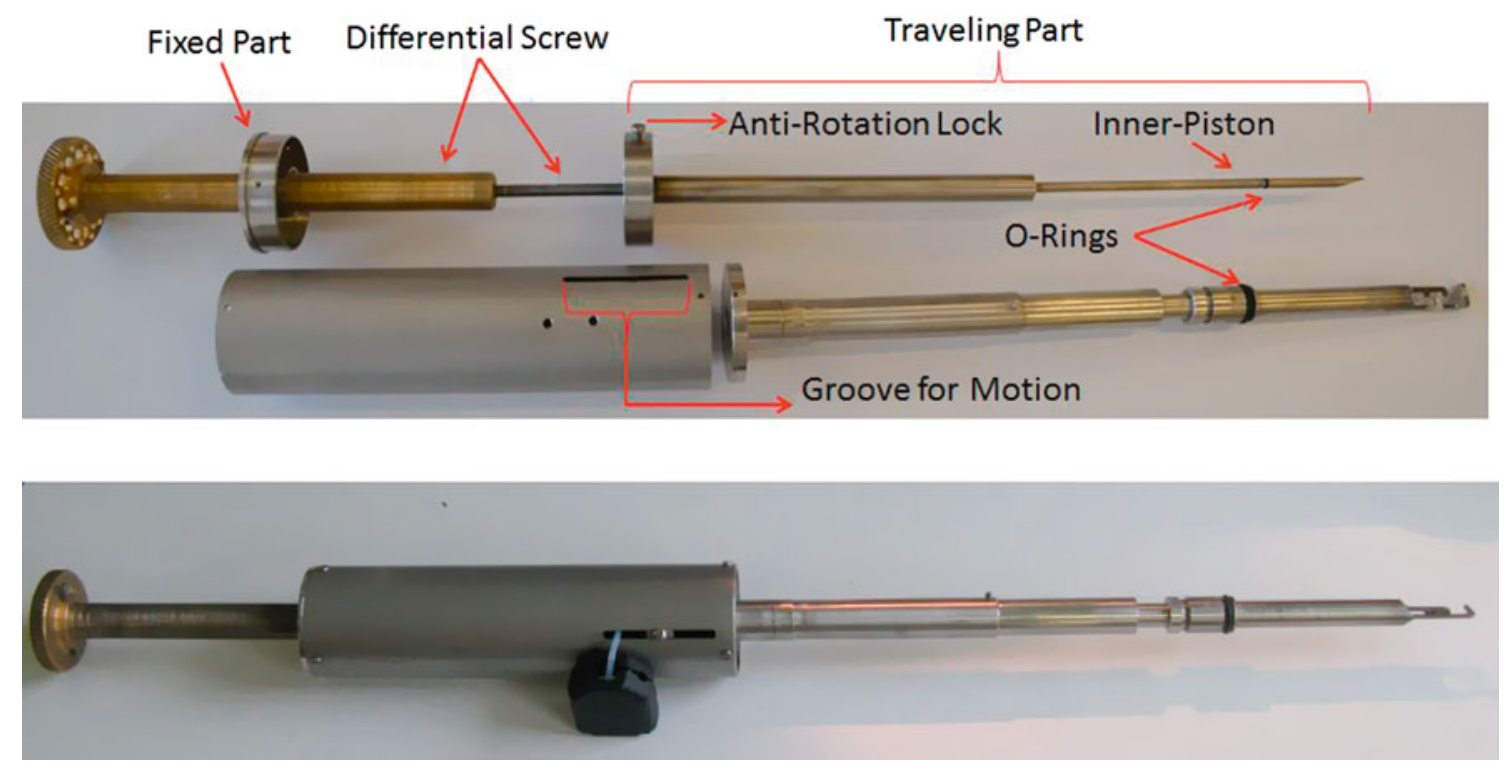

Fig. 2. (A) Disassembled and (B) assembled in situ TEM sample holder.

The differential-screw consists of a shaft with two threads on either ends with different pitches. One of the threads is screwed in to a fixed component while the other thread of the shaft is screwed in to the 'traveling' component. For the differential action to occur, the traveling component should be restricted from rotation. The direction of motion and the exact distance moved by the traveling component is jointly determined by the handedness of the threads and the values of their pitches. For a given amount of rotation of the shaft, the effective displacement of the traveling component is given by the difference in their pitches if they are both like-handed (right-right or left-left) and by the addition of their pitches if they are mixed-handed (right-left).

For the TEM sample-holder described in this article, the pitch of the external screw was $0.8 \mathrm{~mm}$ and that of the inner screw was $1 \mathrm{~mm}$. They were both right-handed. With this set-up, when the external rod is rotated by $2 \pi$ in clock-wise direction, the inner-piston is withdrawn by $200 \mu \mathrm{m}$, thereby relaxing the sample. Conversely, when the external-screw is rotated in counter clock-wise, the inner-piston is advanced, thereby flexing the sample. In this way, the positioning of the inner-piston was controlled to within tens of $\mu \mathrm{m}$ precision thereby finely controlling the degree of flexion of the sample.

\section{Results}

In this section we illustrate a successful demonstration of the in situ holder. A gold wire was suspended on a flexible Kapton ${ }^{\circledR}$ substrate using conventional low vapor-pressure resin epoxy TorrSeal from Varian Inc. The schematic corresponding to the configuration is shown in Figure 1C. The substrate-wire ensemble was then manually mounted between the forks and inserted in the microscope air-lock chamber. Since the in situ holder has more empty space than a conventional sample holder, there is more than normal amount of air to be pumped out. This was achieved by few cycles of roughing-pump while parked in the air-lock section of the microscope. The holder was subsequently introduced into the column of the microscope, between the pole-pieces. The electron gun was turned on once an acceptable level of vacuum $\left(5 \times 10^{-7}\right.$ torr $)$ was achieved, normally within $15-20 \mathrm{~min}$.

Figure 3 shows a sequence of bright-field (BF) images of gold wire at various stages of rupture induced by flexing the substrate using the in situ holder. It can be noticed that there is considerable amount of plastic deformation before the final rupture. It can also be seen that the final neck dimension before fracture is an order of magnitude smaller than the original diameter.

This demonstrates a successful application of the in situ holder developed in this work. This can, of course, be extended to study electronic properties of nanowires by passing current through the sample simultaneously and evaluating the resistance.

\section{Other considerations}

In this section, we discuss the various technical problems encountered during this work. These considerations must be borne in mind during future development of similar instruments. Because of the nature of the set-up, troubleshooting is not very straight forward and therefore, we believe this discussion will be helpful in laying out the possible causes of any malfunctioning.

The material used for sample holder fabrication has to meet several criteria. Firstly and most importantly, it has to be a non-magnetic material. At least the portion inside the column has to be from non-magnetic material, otherwise the sample-holder will get stuck to the objective-lens 

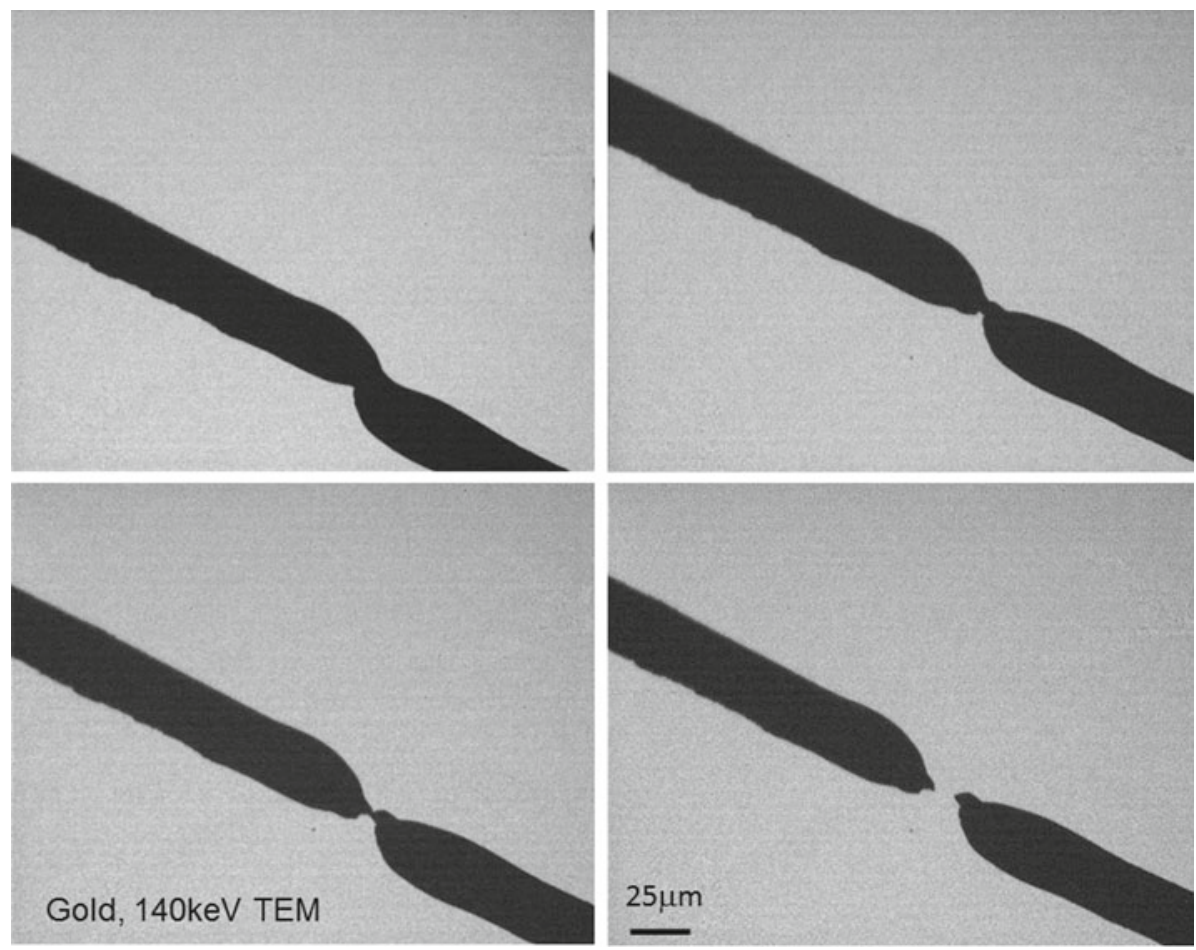

Fig. 3. Sequence of BF images of gold wire taken during various stages of rupture caused by flexing the substrate using the in situ TEM holder.

pole pieces. Secondly, the material has to be sufficiently light-weight such that the whole ensemble is light enough to be moved using the sample stage controls of the microscope. This is needed to exactly position the sample on the optic axis of the microscope. In the current demonstration, we used titanium for the whole assembly except the differential-screw and the fixed-part (see Fig. 2A) which are further removed from the column.

Virtual leaks happen when a pocket of air is trapped between low-vacuum chambers and thus cannot be efficiently pumped out. Thus, the vacuum in the microscope column could progressively deteriorate during operation which can result in decreased life of the electron-gun filament. Generally, when the column pressure reaches an unacceptably high level, the microscope will automatically shut-down. Thus, it is important to eliminate possible virtual leaks during the designing as well as manufacturing stages.

Vacuum problems can also be created by scratches made by the sample holder on the holder entrance path of the microscope. To avoid such problems, sharp corners should be rounded-off. This is particularly important at the leading edge of the sample holder consisting of the counteracting forks.

Electrical charging of the insulating Kapton substrate is possible during operation. This can be limited by applying silver paint around the substrate. Another approach is to evaporate discontinuous network of gold on the substrate such that the electrons are conducted away from the substrate region. The charging of the substrate can limit the spatial resolution achievable with the set-up, therefore, care must be taken to fully control this effect.

\section{Conclusion}

An in situ mechanically-controlled break-junction for TEM was designed and developed. The functioning of the holder was successfully demonstrated using a gold wire suspended on a flexible substrate. The technical diagrams are provided along with a detailed discussion on the technical challenges involved and the pitfalls to avoid in developing similar in situ holders.

This study was funded by the Agence Nationale de la Recherche (ANR) project SUD, France

\section{References}

1. H. Saka, T. Kamino, S. Arai, K. Sasaki, MRS Bulletin 33, $93(2008)$

2. M. Adrian, J. Dubochet, J. Lepault, A.W. McDowall, Nature 308, 32 (1984)

3. H. Zheng et al., Nat. Commun. 1, 144 (2010)

4. T. Kasahara, H.S. Park, D. Shindo, H. Yoshikawa, T. Sato, K. Kondo, J. Magnetism Magn. Mater. 305, 165 (2006)

5. S. Giorgio, S. Sao Joao, S. Nitsche, D. Chaudanson, G. Sitja, C.R. Henry, Ultramicros. 106, 503 (2006)

6. E.A. Stach et al., Microsc. Microanal. 7, 507 (2001)

7. H. Ohnishi, Y. Kondo, K. Takayanagi, Nature 395, 780 (1998)

8. M. Kociak, K. Suenaga, K. Hirahara, Y. Saito, T. Nakahira, S. Iijima, Phys. Rev. Lett. 89, 155501 (2002)

9. M. Viret et al., Phys. Rev. B 66, 220401 (2002)

10. T. Taychatanapat, K.I. Bolotin, F. Kuemmeth, D.C. Ralph, Nano Lett. 7, 652 (2007) 